\title{
Evaluation of treatment outcome of implant assisted removable dentures using OHRQoL.
}

CLINICAL

INNOVATIONS

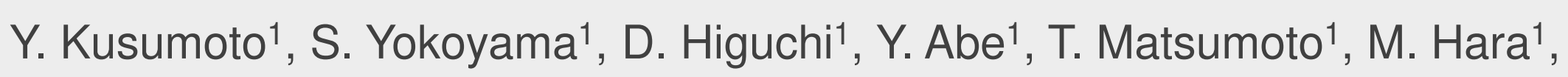

K. Mukawa ${ }^{1}$, Y. Sato ${ }^{2}$, K. Baba ${ }^{1}$

'Showa University School of Dentistry, Tokyo, Japan. ${ }^{2}$ National Defense Medical College, Saitama, Japan.

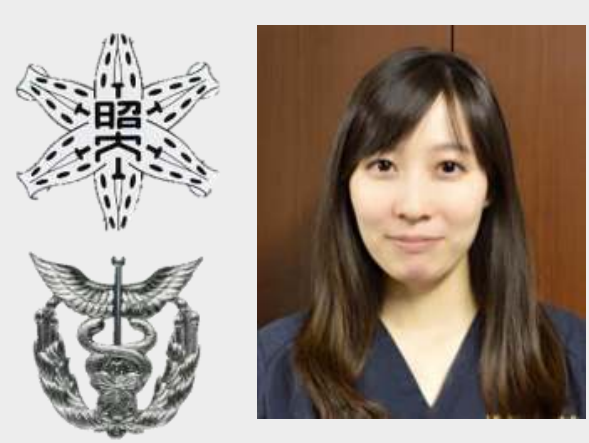

\section{Background and Aim}

It has been well documented that implant supported fixed prostheses (ISFPs) generally provide well treatment outcome. However, the more patients lose their teeth, the more implant placements for ISFPs would be needed and ISFPs are not always applicable to patients with multiple missing teeth due to high cost and invasiveness. Implant assisted removable dentures (IARDs) such as implant overdenture (IOD) or implant assisted removable partial denture (IA-RPD) are alternative treatment option in such cases (Fig. 1). However, there are few studies that evaluated treatment outcome of IARDs. The aim of this study was to compare the treatment outcome of IARDs with that of ISFPs by oral health related quality of life (OHRQoL).

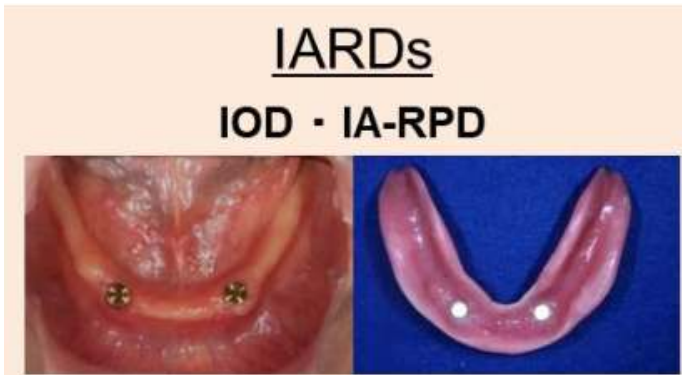

Inexpensive

Less invasive

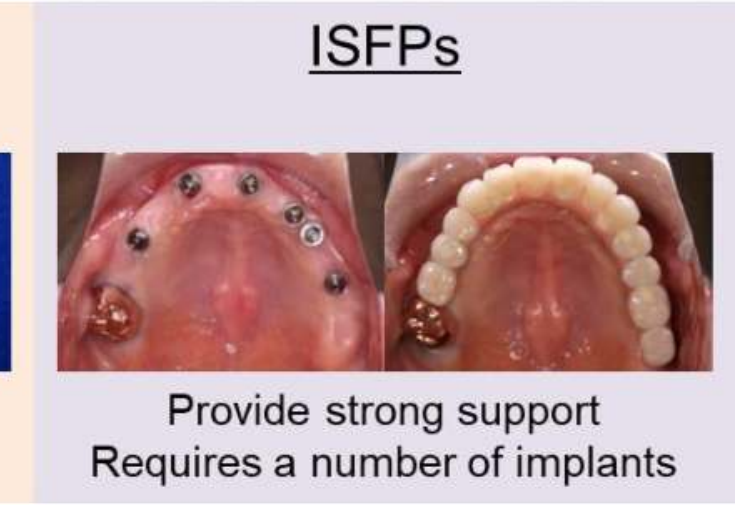

Fig. 1. Characteristic of types of treatments

\section{Methods and Materials}

\section{Subjects}

Subjects were recruited at the department of Prosthodontics and Implant Center at Showa University Dental Hospital between April 2008 and April 2015. In total, 33 patients (65.3 \pm 10.6 years old) participated in this study (Table 1, 2, Fig. 2). The study protocol was approved by the Ethics Committee of Showa University (\#2007-29).

\section{Table 1. Inclusion and exclusion criteria}

Inclusion criteria

- Classified as Eichner B3, B4, C1, C2 and C3.

Exclusion criteria

- Presence of pain in orofacial region.

Implant surgery contraindicated due to general health problem.

Difficulty in answering self-administered questionnaires.

\begin{tabular}{|c|c|c|c|}
\hline$n=33$ & $\begin{array}{c}\text { IARDs } \\
n=18 \\
\text { IOD }: 10, \text { IA-RPD }: 8\end{array}$ & $\begin{array}{c}\text { ISFPs } \\
n=15\end{array}$ & $P$ value \\
\hline Age & $71.2(8.3)$ & $58.1(8.6)$ & $<0.001 *$ \\
\hline Male / Female & $5 / 13$ & $6 / 9$ & 0.46 \\
\hline $\begin{array}{l}\text { Number of missing } \\
\text { teeth }\end{array}$ & $21.0(4.8)$ & $17.5(4.6)$ & $0.040 *$ \\
\hline Number of implant & $2.7(1.6)$ & $7.7(2.4)$ & $<0.001 *$ \\
\hline
\end{tabular}

\section{Measurement of OHRQoL}

OHRQoL was evaluated by using the Japanese version of Oral Health Impact Profile (OHIP-J) 1), which was administered before (baseline) and after delivering the prostheses (post-treatment). The OHIP summary score as well as 4 sub-domains, which included "Oral Function", "Orofacial Pain", "Orofacial Appearance", and "Psychosocial Impact" were calculated (Fig. 3) ${ }^{2)}$.

Oral Function

\section{Oral Health Impact Profile}

\section{Fig. 3. OHIP 4 sub-domains of Oral Health Impact Profile}

\section{Statistical analyses}

The 4 sub-domain scores were standardized as percentage of the full score for each sub-domain.

T-test, Paired t-test, JMP Pro 13.0.

With the probability set at the $5 \%$.

\section{Results}

\section{I . Improvements in OHIP scores for each type of treatment}

The average baseline and posttreatment OHIP summary scores for IARDs were $79.2 \pm 40.8$ and $36.8 \pm 25.6$ and those for ISFPs were $83.4 \pm 24.8$ and $33.7 \pm 22.1$, respectively. These scores were significantly improved by both types of the treatment $(P<0.001$, Fig. 4). All of 4 sub-domain scores were also significantly improved by both types of the treatment $(P<0.05$, Fig.

Baseline $\longrightarrow$ Post-treatment $*: \mathrm{P}<0.05$

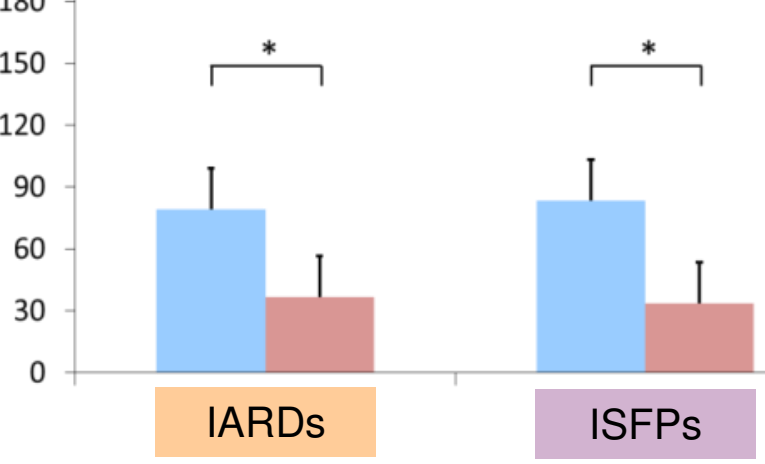

Fig. 4. The average OHIP summary score or IARDs and ISFPs $5)$.

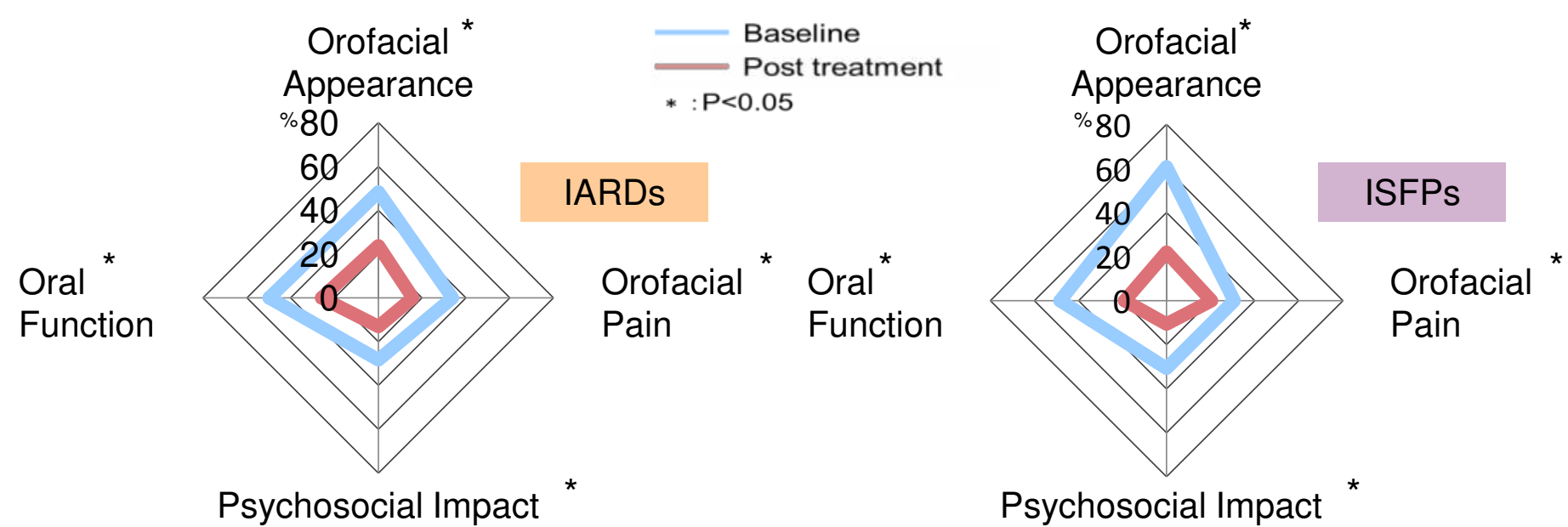

Fig. 5. The percentages of the 4 sub-domain scores for IARDs and ISFPs

\section{Baseline and post-treatment OHIP scores}

No significant difference was found in the summary score and 4 sub-domain scores $(P>0.05$, Fig. 6,7$)$.

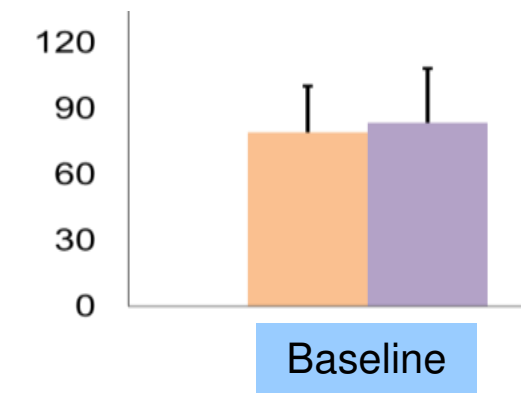

Fig. 6. The average OHIP summary score at baseline and post treatment

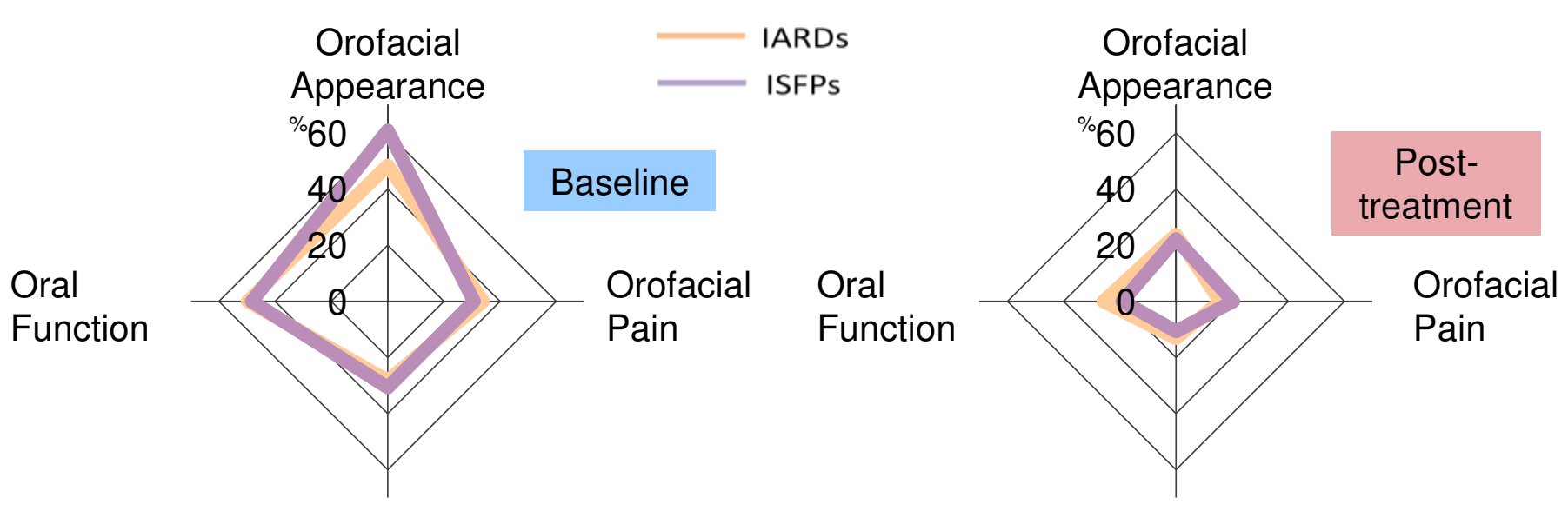

Psychosocial Impact

Psychosocial Impact

Fig. 7. The percentages of the 4 sub-domain scores at baseline and post treatment

\section{Conclusions}

This study revealed that both IARDs and ISFPs had significant effects on the improvement in OHRQoL. Besides, the effects of the types of the treatment on the magnitude of OHRQoL improvements and the posttreatment OHRQoL status resulted to be not significant. Within the limitations of this study, which includes the small sample size and demographic differences, these results suggest that IARDs are inexpensive and less invasive treatment option for patients with multiple missing teeth that allows acceptable treatment outcome.

\section{References}

1) Yamazaki M, Inukai M, Baba K, John MT. Japanese version of the Oral Health Impact Profile (OHIP-J). J Oral Rehabil 2007; 34:159-68.

2) John MT, Reissmann DR, Feuerstahler L, Waller N, Baba K, Larsson P, et al. Exploratory factor analysis of the Oral Health Impact Profile. J Oral Rehabil 2014; 41: 635-643. 